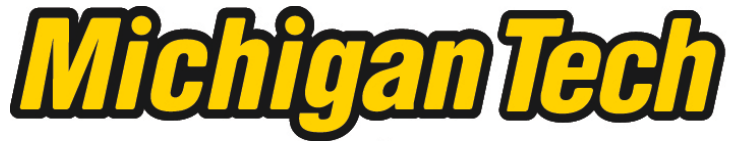 \\ Michigan Technological University Create the Future Digital Commons @ Michigan Tech
}

Dissertations, Master's Theses and Master's Reports - Open

Dissertations, Master's Theses and Master's

Reports

2010

\section{Measuring the elastic modulus of polymers using the atomic force microscope}

Daniel Hoffman

Michigan Technological University

Follow this and additional works at: https://digitalcommons.mtu.edu/etds

Part of the Engineering Science and Materials Commons

Copyright 2010 Daniel Hoffman

\section{Recommended Citation}

Hoffman, Daniel, "Measuring the elastic modulus of polymers using the atomic force microscope", Master's Thesis, Michigan Technological University, 2010.

https://doi.org/10.37099/mtu.dc.etds/27

Follow this and additional works at: https://digitalcommons.mtu.edu/etds

Part of the Engineering Science and Materials Commons 


\title{
Measuring the Elastic Modulus of Polymers using the Atomic Force
} Microscope

\author{
By
}

Daniel Hoffman

\begin{abstract}
A THESIS
Submitted in partial fulfillment for the degree of

MASTER OF SCIENCE IN

MATERIALS SCIENCE AND ENGINEERING
\end{abstract}

MICHIGAL TECHNOLOGICAL UNIVERSITY

2010

(C) 2010 Dan Hoffman 
This thesis, "Measuring the Elastic Modulus of Polymers using the Atomic Force Microscope," is hereby approved in partial fulfillment of the requirements for the Degree of MASTER OF SCIENCE IN MATERIALS SCIENCE AND ENGINEERING.

Department of Materials Science and Engineering

Signatures:

Thesis Advisor

Jaroslaw Drelich

Department Chair

Mark Plichta

Date 


\section{Table of Contents}

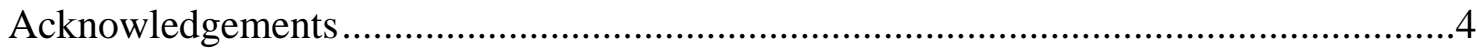

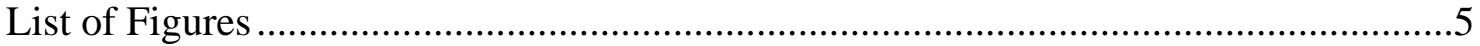

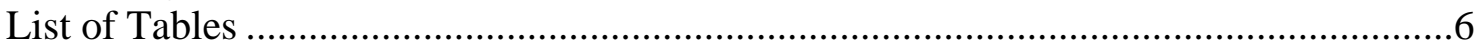

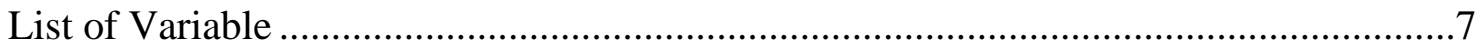

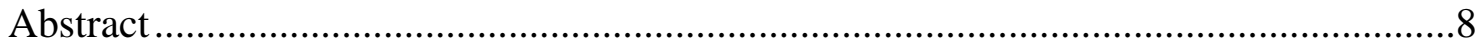

1. Introduction

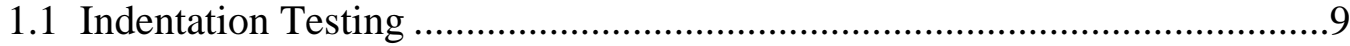

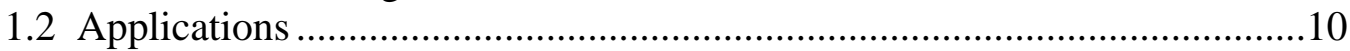

2. Background

2.1 Depth Sensing Indentation..................................................................11

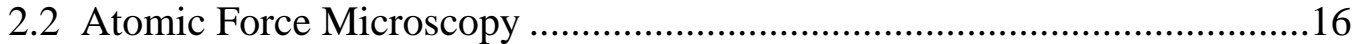

2.3 AFM Nanoindentation .......................................................................19

2.4 Comparison of the Techniques ...........................................................2 21

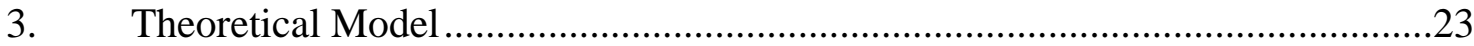

4. $\quad$ Experimental Procedure

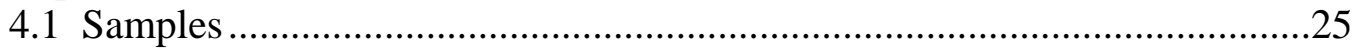

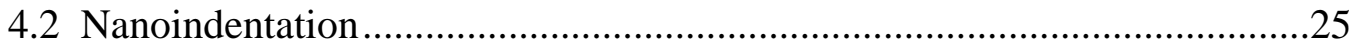

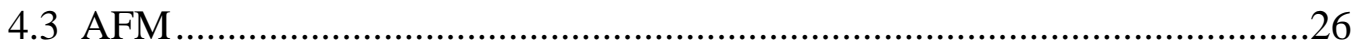

5. Results and Discussion

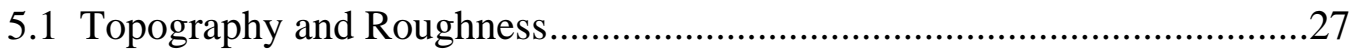

5.2 Mechanical Properties from Nanoindenter ..............................................31

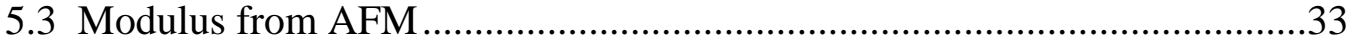

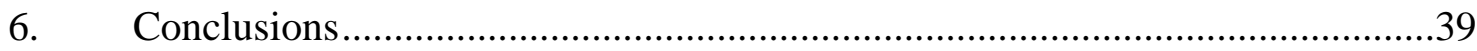

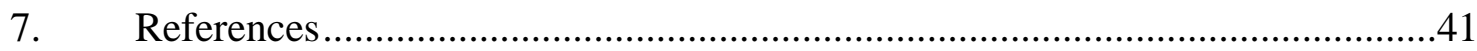




\section{Acknowledgments}

I would like to say thank you to my advisor Dr. Jaroslaw Drelich for helping with my thesis, and Dr. Ibrahim Miskioglu for helping with running nanoindentation testing. 


\section{List of Figures}

Figure 1: (a) Typical load verses displacement curve from a nanoindentation experiment. (b) A schematic of indentation geometry at the maximum and final load positions

Figure 2: A model of a Berkovich indenter tip. The face angle "a" is approximately $65.3^{\circ}$.

Figure 3: The Dimension 3000 atomic force microscope used for this test.

Figure 4: An image of a typical single beam AFM cantilever and schematic of a typical AFM set up.

Figure 5: The top image shows a schematic of the AFM cantilever operating in contact mode. The bottom shows the same image but with the AFM in tapping mode.

Figure 6: The graph on the left shows a voltage versus displacement curve that would be output by the AFM from a nanoindentation test. The figure on the right shows the same curve converted into a force curve.

Figure 7: Topographic images of each sample taken prior to nanoindentation.

Figure 8: Cross sectional images of the sample surfaces.

Figure 9: A sample loading curve showing the load schematic for the nanoindentation tests.

Figure 10: Representative distance verses deflection curves from the nanoindentation tests on the AFM.

Figure 11: FESEM images of the tip used in the second set of tests. 


\section{List of Tables}

Table 1: Average roughness data taken from five images.

Table 2: Elastic modulus data from both the nanoindentation tests and from databases for comparison. The Poisson's ratio found for each sample is also given.

Table 3: Average K-values measured from fifteen unloading curves for each sample.

Table 4: Comparison of the results from the nanoindenter and the AFM.

Table 5: T-statistics for each of the samples compared with the critical t-value for a 95\% confidence limit. 


\section{List of Variables}

P Load during indentation

$\mathrm{h} \quad$ Dispacment during indentation

$\alpha_{0} \quad$ Constant relating load and displacement

$\mathrm{m} \quad$ Constant relating load and displacement determined by tip geometry

$\mathrm{h}_{\mathrm{f}} \quad$ Final unloading depth during indentation

S Contact stiffness

a Contact radius

$\mathrm{A}_{0} \quad$ Projected area of the elastic constant

$\mathrm{E}_{\mathrm{r}} \quad$ Reduced elastic modulus

$\mathrm{E}_{\mathrm{s}} \quad$ Elastic modulus of the sample

$\mathrm{E}_{\mathrm{i}} \quad$ Elastic modulus of the indenter

$v_{\mathrm{s}} \quad$ Poisson's ratio of the sample

$v_{i} \quad$ Poisson's ratio of the indenter

$\mathrm{h}_{\max } \quad$ Maximum depth of indentation

$\mathrm{h}_{\mathrm{c}} \quad$ Contact depth during indentation

C Total compliance

$\mathrm{C}_{\mathrm{f}} \quad$ Load frame compliance

$\mathrm{C}_{\mathrm{s}} \quad$ Sample compliance

$\delta \quad$ Vertical tip deflection

D Electrical signal

A Cantilever sensitivity

$\mathrm{D}_{0} \quad$ Initial deflection signal

$\mathrm{k} \quad$ Cantilever spring constant

$\delta \quad$ Vertical change of the piezo actuator

$\mathrm{K} \quad$ Slope of a $\delta$ verses D unloading curve

$\alpha \quad$ Cantilever tip property 


\begin{abstract}
Testing a new method of nanoindentation using the atomic force microscope (AFM) was the purpose of this research. Nanoindentation is a useful technique to study the properties of materials on the sub-micron scale. The AFM has been used as a nanoindenter previously; however several parameters needed to obtain accurate results, including tip radius and cantilever sensitivity, can be difficult to determine. To solve this problem, a new method to determine the elastic modulus of a material using the atomic force microscope (AFM) has been proposed by Tang et al. This method models the cantilever and the sample as two springs in a series. The ratio of the cantilever spring constant $(k)$ to diameter of the tip (2a) is treated in the model as one parameter $(\alpha=k / 2 a)$. The value of $a$, along with the cantilever sensitivity, are determined on two reference samples with known mechanical properties and then used to find the elastic modulus of an unknown sample. To determine the reliability and accuracy of this technique, it was tested on several polymers. Traditional depth-sensing nanoindentation was preformed for comparison. The elastic modulus values from the AFM were shown to be statistically similar to the nanoindenter results for three of the five samples tested.
\end{abstract}




\section{Introduction}

\subsection{Indentation Testing}

The purpose of this research was to test a newly proposed method of nanoindentation with the atomic force microscope (AFM) to determine the elastic modulus of polymer samples. Nanotechnology has become a major field in science and engineering with products ranging from cosmetics, auto parts, and electronics using nano-scale component. Polymers are frequently used in composites, thin films, or nanoparticles, and it has been shown that the local properties in these components can differ from their bulk properties [1, 2, 3].

Indentation testing is a simple and convenient way to measure the properties of a material. The hardness and elastic modulus are the two most common properties measured by indentation testing. Depth sensing indentation (DSI) involves pushing an indenter tip into a material and measuring the load versus displacement, which can then be used to find the desired property of the sample. Rockwell and Vicker's hardness testers are common instruments used for this purpose; however, they are not effective for use on nanomaterials or thin films due to the size of the indenters and the depth of penetration. To facilitate more accurate study of a material's properties on a sub-micron scale, many nanoindentation methods have been developed [4, 5, 6].

These experiments in nanoindentation have typically used a purpose built instrument; however, the AFM can also be used for mechanical testing. While its primary function is to image the surface of a sample, the AFM has been employed as a nanoindenter $[7,8,9]$. Most AFM methods have used the same principles as traditional nanoindentation. To obtain accurate results, several properties of the AFM cantilever 
must be known, particularly the spring constant, sensitivity, and tip radius. These properties can be difficult to determine; however, a new technique has been proposed that can calculate these properties by performing tests on reference samples [10]. This technique was tested in this MS program on several polymer materials to determine its accuracy and reliability.

\subsection{Applications}

Improving nanoindentation techniques will allow for more accurate characterization of materials at the sub-micron scale. This will benefit the many industries that now use nanocomponents in their products. The interfacial region of composites could be studied more accurately. This region has been shown to have an important effect on the overall performance of the composite [11, 12]. Using lower loads and indentation depths, AFM indentation could be used to study thin films while avoiding the effects of the underlying substrate [2]. This new method could make characterizing the nano-scale properties of materials more convenient and precise, aiding in further advancements in chemistry, biology, and materials science [13]. 


\section{$2 \quad$ Background}

\subsection{Depth Sensing Indentation}

Depth sensing indentation (DSI) tests involve pushing an indenter tip into a sample and measuring the load and displacement of the tip in order to determine mechanical properties of the sample [14]. The most common properties to be measured are the hardness and elastic modulus, which can be calculated using contact mechanics and the tip-sample contact area $[5,15]$. In order to accurately calculate the properties of the material, the tip shape and contact area must be known and the data must be corrected for load frame compliance, as well as problems with the sample such as creep, thermal drift, which is caused by changes in ambient temperature or the equipment heating, and pile up, which can be caused by plastic deformation in the sample [15, 16]. Typically, the indentation depths produced by this method fall in the sub-micron or even nano-scale range. Due to these low depths, DSI is commonly referred to as nano-indentation. This method has become an important tool for materials characterization. The low depths allow it to be used on thin films, avoiding interference from the substrate below. In addition, it can be used on small components for electronics, measuring properties of individual grains of materials, or any other application where localized small scale testing is needed [15].

Methods for determining the mechanical properties of a material are derived from classic contact mechanics equations, such as the Hertz model which deals with the elastic contact between two materials [10]. The relationship between the load, depth, and contact area were then derived by Sneddon [17]. His equation gives a simple relationship between the load and displacement for simple tip geometries: 


$$
P=\alpha_{0} h^{m}
$$

where $\mathrm{P}$ is the load, $\mathrm{h}$ is the displacement, and $\alpha_{0}$ and $\mathrm{m}$ are constants. The parameter " $\mathrm{m}$ " is determined by the geometry of the tip; for a cylinder $\mathrm{m}=1$, and for a cone $\mathrm{m}=2$ [5]. This model was expanded on by Oliver and Pharr [5] to include the final unloading depth, $\mathrm{h}_{\mathrm{f}}$ :

$$
P=\alpha_{0}\left(h-h_{f}\right)^{m}
$$

As the indenter pushes into the sample, the load and displacement are continuously measured, producing a load versus displacement curve shown in Figure 1a. The displacement values are directly related to the indentation depth as seen in the schematic shown in Figure 1b. Equation 2 models the unloading curve by a power law function, and it was realized that the derivative of this curve would model the contact between the tip and sample at that point, which could then be used to determine mechanical properties $[5,14]$. The slope at the point of maximum load is defined as the contact stiffness, S, which is modeled using Sneddon's solution for elastic contact:

$$
S=2 a E_{r}=\frac{2}{\sqrt{\pi}} E_{r} \sqrt{A_{o}}
$$

where "a" is the contact radius, $A_{0}$ is the projected area of the elastic contact, and $E_{\mathrm{r}}$ is the reduced modulus. The elastic modulus of the sample material can then be calculated by using the reduced modulus:

$$
\frac{1}{E_{r}}=\frac{\left(1-v_{s}\right)}{E_{s}}+\frac{\left(1-v_{i}\right)}{E_{i}}
$$

where $v_{s}$ and $E_{s}$ are the Poisson's ratio and elastic modulus of the sample, respectively, and $v_{\mathrm{i}}$ and $E_{\mathrm{i}}$ are the Poisson's ratio and elastic modulus of the indenter, respectively. 
Thus, if the properties of the indenter are known, and the Poisson's ratio of the sample is known or can be reasonably estimated, then the elastic modulus of the sample can be calculated.

These models assume ideal conditions during the indentation process, but this normally does not occur in practice. Two important factors that need to be accounted for are the load frame compliance and the tip area function $[5,15]$. The total depth reading can include deformations in the indenter as well as the sample, which is termed the load frame compliance. For an accurate measurement, this must be factored out of the indentation depth. While the general shape of the tip is known, the geometry is usually not ideal and therefore must also be adjusted. In addition to developing the power law rule used to determine the elastic modulus, Oliver and Pharr also developed methods to determine the load frame compliance and the tip area function [5].

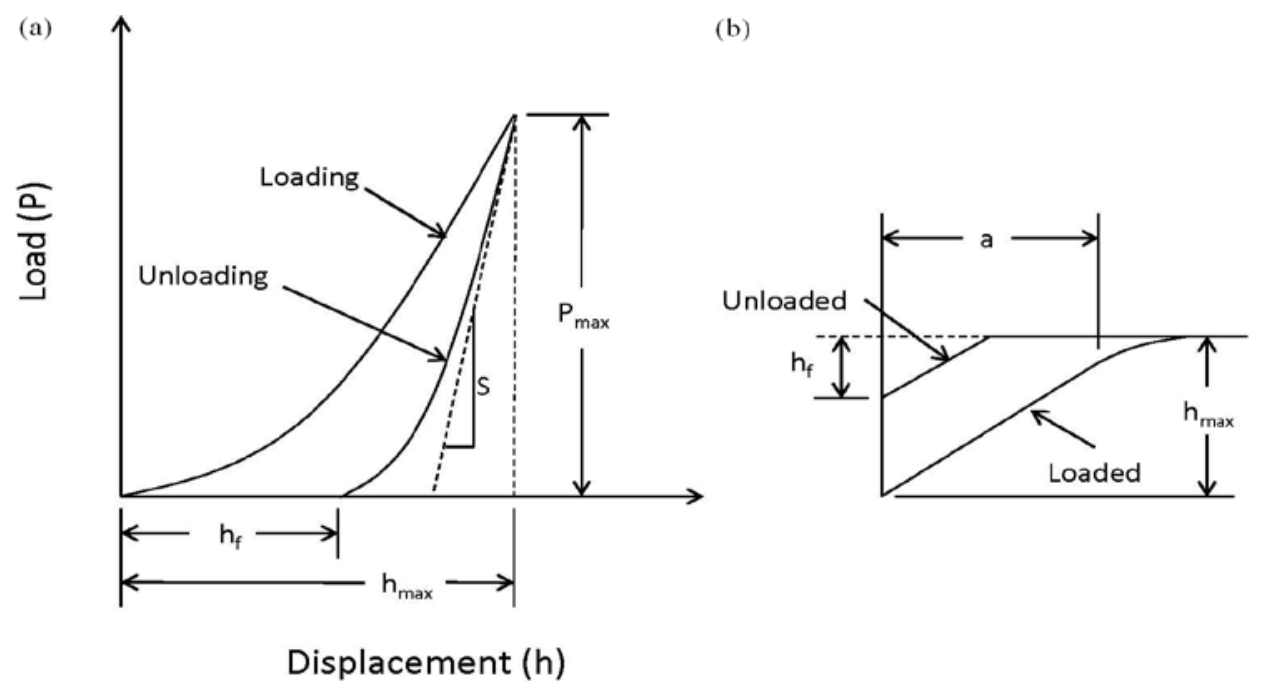

Figure 1: (a) Typical load verses displacement curve from a nano-indentation experiment. $h_{\max }$ is the maximum depth of the indentation, and $h_{f}$ is the final depth after elastic recovery. (b) A schematic of indentation geometry at the maximum and final load positions. $h_{c}$ is the contact depth. 
Their method assumes that the elastic modulus is independent of indentation depth and the load frame and sample can be modeled as two springs in a series. Thus the total compliance, $\mathrm{C}$, is the sum of the sample compliance, $\mathrm{C}_{\mathrm{s}}$, and the load frame compliance, $\mathrm{C}_{\mathrm{f}}$ :

$$
C=C_{f}+C_{s}
$$

The sample compliance is defined as the inverse of the stiffness (Equation 3), and the total compliance is the slope of the load versus displacement curve; thus the equation becomes:

$$
\frac{\mathrm{d} P}{\mathrm{~d} h}=C_{f}+\frac{\sqrt{\pi}}{2 E_{r}} \frac{1}{\sqrt{A_{0}}}
$$

Using this analysis, Oliver and Pharr [5] developed a technique to calculate both the load frame compliance and the tip area function using a single reference test and solving Equation 6 for $C_{f}$ and $A$. While this method can be effective, generally a less math intensive method is used [14]. In this method, the load frame compliance is found by testing a reference sample and finding the value for the intercept of Equataion 6. For this calculation, the tip area $\mathrm{A}$ is taken to be an ideal geometry, which was given a value of 24.5 $\mathrm{h}_{\mathrm{c}}{ }^{2}$ by Oliver and Pharr [5]; thus Equation 6 can be rewritten as [15]:

$$
\frac{\mathrm{d} h}{\mathrm{~d} P}=C_{f}+\frac{\sqrt{\pi}}{2 E_{r}} \frac{1}{\sqrt{24.5}} \frac{1}{h_{c}}
$$

In addition to the load frame compliance, the tip area function is also calibrated to account for non-ideal tip geometry. A common tip used in nanoindentation is the Berkovich indenter tip, which has a three sided pyramid shape; a model can be seen in Figure 2. Ideally the indenter could be infinitely sharp, but in practice the tip has some 
degree of rounding, and calibrating the tip area function accounts for this effect. The calibration is made by indenting a reference material and determining the value of $\mathrm{A}$ as a function of $h_{c}$. The tip area function, $A_{0}\left(h_{c}\right)$, takes the form:

$$
A_{0}\left(h_{c}\right)=24.5 h_{c}^{2}+B_{1} h_{c}+B_{2} h_{c}^{1 / 2}+B_{3} h_{c}^{1 / 4}+. .
$$

As well the compliance and tip area function, other material related issues can introduce error into the results. Creep can occur within the material while under load. This can cause the indentation depth to increase with time. To account for this effect, a hold segment can be added at full load during which the system measures the creep rate [10]. Changes in temperature during testing can cause expansion or contraction in the sample or indenter which can cause changes in the contact area [15]. This can be accounted for by adding a hold period at low load after the indentation during which the system can again determine and account for the drift rate [10].

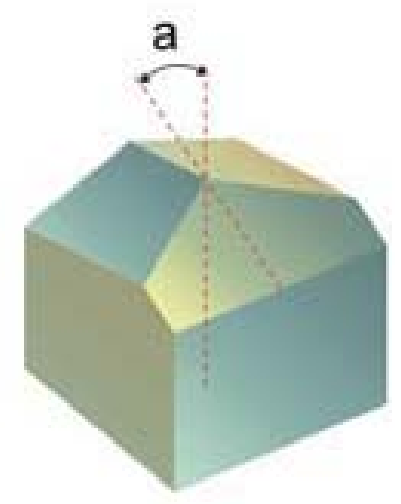

Figure 2: A model of a Berkovich indenter tip. The face angle "a" is approximately $65.3^{\circ}$. 
Sink-in and pile-up effects are also important material related issues that can occur with certain materials. The Oliver and Pharr method as described can account for sink-in effects, but pile-up can produce error in the contact area and so affect the final result [18]. Pile-up occurs when deformation in the sample displaces material to the edge of the indentation. This material supports the indenter and increases the contact area [15, 19]. If pile-up occurs, the Oliver and Pharr method underestimates the contact area, A, which in turn causes an increase in the estimated elastic modulus or hardness [18]. Sinkin and pile-up effects can be accounted for by measuring the contact area using either scanning electron microscopy or atomic force microscopy to image the indentation.

\subsection{Atomic Force Microscopy}

The AFM belongs to a family of instruments called scanning probe microscopes (SPM). Binning and Rohrer et. al. developed the first SPM in 1982. It was used to obtain atomic scale topographic images by placing a conducting tip near the sample and measuring tunneling current between the tip and the sample [20]. Binning and Rohrer expanded on this technique with the invention of the AFM in 1986 [21]. The AFM was first used to scan the topography of the sample, and can achieve resolutions down to nanometer scale. In addition to topography, the AFM can also perform nanoindentation. Using the AFM as a nanoindenter allows the surface of the sample to be scanned and characterized then indented with the same tip. This way a suitable area can be found to test, avoiding roughness that can decrease the accuracy of the results, or particular features of interest can be located and tested. 


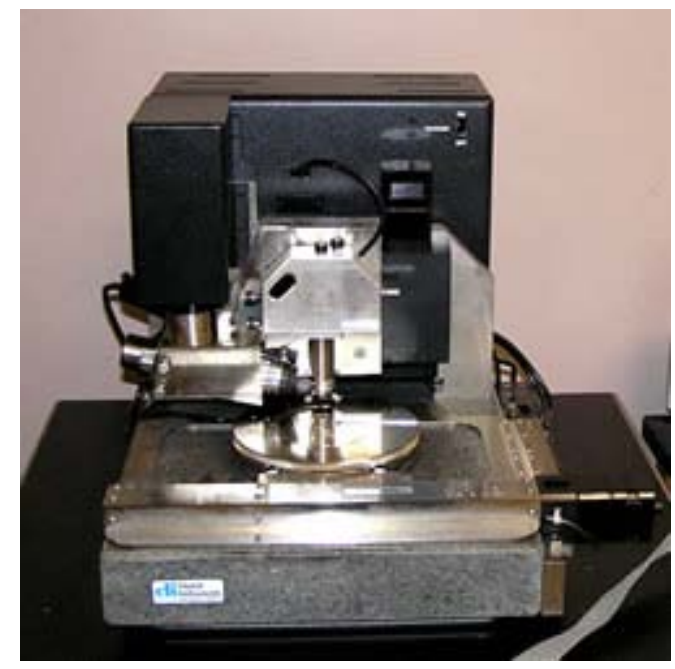

Figure 3: The Dimension 3000 atomic force microscope that was used for this test.

The AFM operates by using a probe to "feel" the surface of the sample. A typical probe consists of three parts: a chip, a cantilever, and a tip. The chip provides a base to mount the probe into the AFM. The cantilever extends out from the chip, and has a sharp tip on the end. The cantilever can have either a single beam or double beam design, depending on the application. In this paper, only single beam cantilevers were used. Probes are commonly made of silicon and typically have tip radius of $5-10 \mathrm{~nm}$ and a spring constant between $20-100 \mathrm{~N} / \mathrm{m}$ [21]. Figure 4 shows an image of a single beam cantilever and tip.

To translate the mechanical motion of the cantilever to usable data, a laser and photodiode system is used. A laser is focused on the end of the cantilever where it is reflected into a set of photodiodes. The photodiode array consists of four quadrants, each of which outputs a voltage when the laser hits it. The change in voltage between the diodes is measured and converted into topographic data. The position of the probe is controlled by piezoelectric scanners which can move the indenter in three dimensions 
with nanometer scale resolution. A schematic of a typical AFM system can be seen in Figure 4 .

The AFM has several modes of operation, each of which can produce data on several surface properties. The two most common modes which will be discussed are contact mode and tapping mode. Contact mode was the first mode of operation created for the AFM. In this mode, the tip is put into contact with the sample and dragged over the scan area. As the tip comes into contact with changing topography, the cantilever deflects, which in turn causes the laser to move on the photodiode, outputting topographic data. Contact mode has the advantage of being capable of quickly imaging a sample. However the continuous contact can cause damage to softer samples. To solve this problem a second operating mode, called tapping mode was developed.

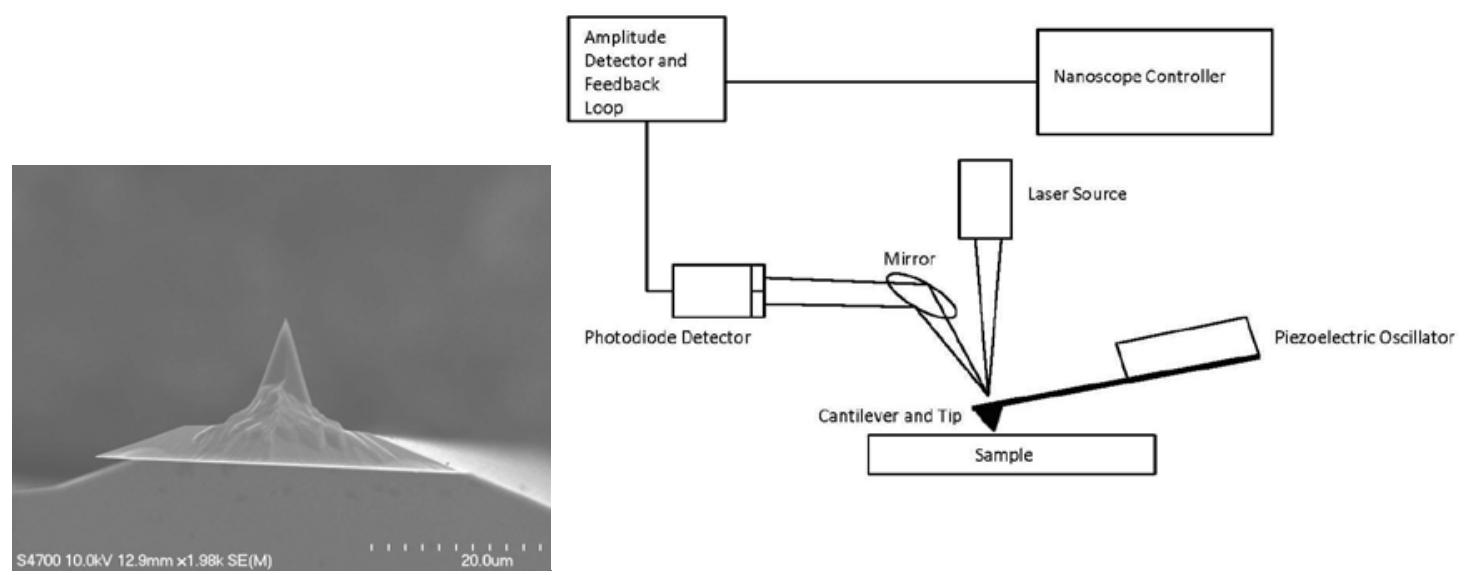

Figure 4: An image of a typical single beam AFM cantilever and schematic of a typical AFM set up. The piezoelectric oscillator controls the tip movement. A laser is aimed at the end of the cantilever, where the most deflection occurs, and is reflected into the detector. The signal from the detector is processed into topographic data and used to control the tip movement. 

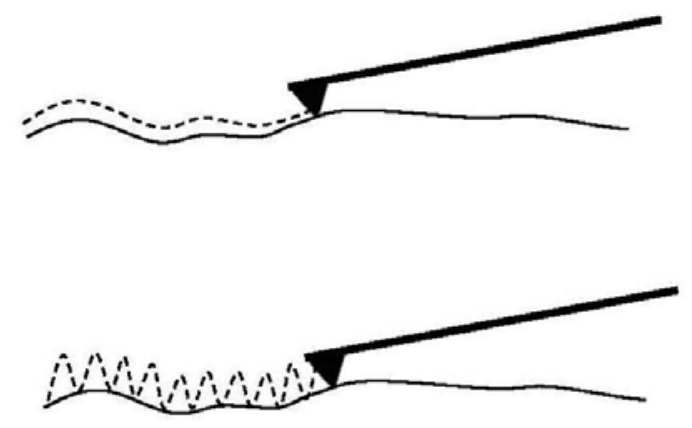

Figure 5: The top image shows a schematic of the AFM cantilever operating in contact mode. The solid line represents the sample surface while the dotted line traces the tip position as the tip moves from left to right. The bottom shows the same image but with the AFM operating in tapping mode.

In tapping mode the cantilever is oscillated above the surface then lowered until it makes contact. In this mode the tip repeatedly taps the surface, rather than remaining in constant contact. The computer records the voltage from the oscillating tip, measuring the amplitude. To operate in tapping mode, the tip is oscillated at its resonant frequency and taps the surface at the bottom of its swing. Once the amplitude is set, the computer will maintain this amplitude by changing the height of the scanner as the tip taps features with varying height. The height at each point is recorded and converted into an image.

\subsection{AFM Nanoindentation}

In addition to topographic information, the AFM can be used as a nanoindenter to obtain data regarding the mechanical properties of the sample. To perform nanoindentation the AFM is operated in force mode. In force mode, the tip is brought into contact with the surface, pushed to a maximum load, and then withdrawn. The voltage on the photodiode is recorded throughout the motion and plotted against the vertical distance to the sample, as seen in Figure 6. By modeling the cantilever as a 
spring, the voltages recorded can be converted into forces by Hooke’s law. Because of this relation, these voltage versus distance curves are commonly called force curves.

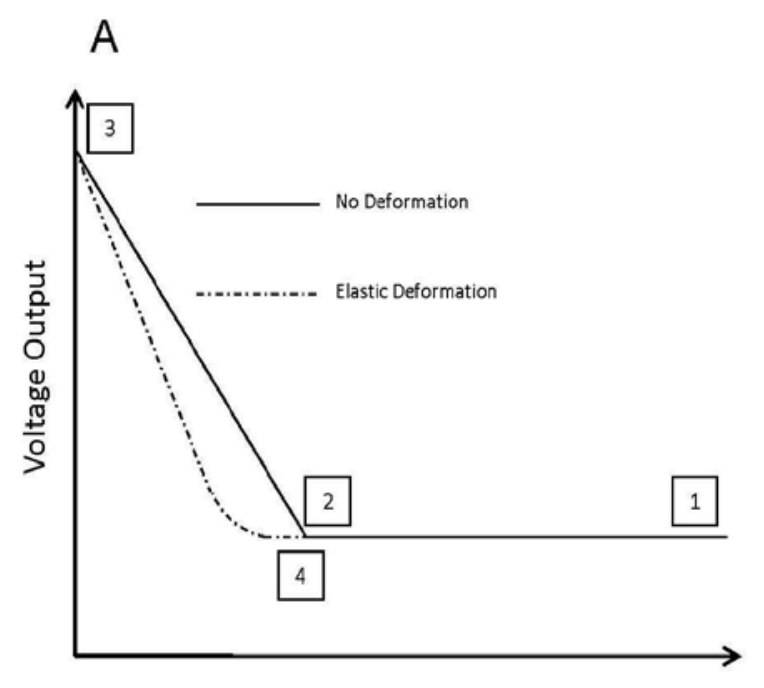

Piezoelectric Actuator Displacement

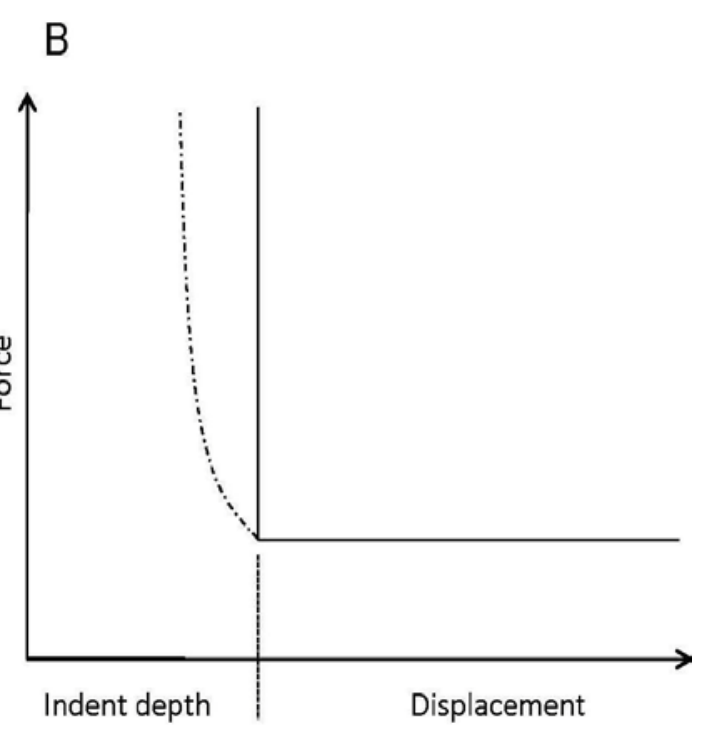

Figure 6: The graph on the left shows a voltage versus displacement curve that would be output by the AFM from a nanoindentation test. Two curves are shown: one with no deformation, which shows a sharp change in slope, and one with elastic deformation, which has a gradual increase in slope caused by the changing contact area between the tip and sample. The figure on the right shows the same curves converted into a force curve.

Figure 6 shows a generic force curve that would result from this mode of testing. The tip begins at a position above the sample, marked as point 1 , and is lowered toward the surface. At point 2 the tip comes into contact with the surface. As the tip is pushed into the sample the cantilever deflects, causing an increase in the voltage. At point 3 the maximum load is reached and the tip retracts. If the contact is purely elastic, the retracting curve will be equal to the approaching curve. If plastic deformation occurred, the retracting curve will trace beneath the approaching curve. The difference between the approach curve and the retracting curve is related to the amount of plastic deformation 
experienced by the sample. A stiff sample will produce a curve close to parallel with the approach curve, while a softer sample will show greater variation.

By converting the voltage versus displacement curve into a force curve, the load and depth data can be obtained. The area function can be determined by rescanning the topography of the indent and measuring it using the software's cross sectional analysis. The Oliver and Pharr method can be used in the same way as in traditional DSI testing [9, 14]. To obtain accurate data from these models, the cantilever sensitivity and tip radius must be known. The cantilever sensitivity is typically calibrated by measuring the slope of the deflection versus displacement curve after indenting a hard sample.

\subsection{Comparison of the Techniques}

The nanoindenter provides a relatively simple method for performing indentation testing. Since the tip shape is well characterized and the test parameters such as load and time are known, computer software can perform the necessary calculations to determine the hardness and elastic modulus. A major disadvantage of this technique is that the sample surface cannot be scanned at high resolutions; it must be removed and imaged with a scanning electron microscope or AFM to fully characterize the surface. This also means that testing nanostructures or phases would be difficult since they could not be located with only the nanoindenter's optical microscope.

The AFM has the advantage of being capable of imaging and indenting the surface with the same probe, thus eliminating the need to transfer samples to a different instrument for surface characterization. The surface scanning features of the AFM allow for particular features or phases to be located and tested accurately. However, the test parameters are more difficult to control in AFM nanoindentation. The properties of the 
probe, such as spring constant, sensitivity, and tip radius need to be known to obtain accurate results. The spring constant can be found using the AFM software, and the cantilever sensitivity can be calibrated; however, estimating the tip geometry can be difficult due to the small size of AFM tips. Scanning electron microscope images or blind reconstruction can be used to estimate the geometry, but can be difficult with small tips [22, 23]. Recently, a new method has been proposed that uses two samples with known properties to calibrate the AFM [10]. The two reference materials are used to calculate the cantilever sensitivity and a new parameter, the cantilever-tip property. Then the modulus of a third unknown material can be measured, reducing the need to estimate cantilever sensitivity or tip geometry. 


\section{Theoretical Method}

The AFM force curves were used to obtain the Young's Modulus of several polymers. This approach models the interaction between the tip and sample as two springs in a series. The small indentation depths and low loads create an elastic interaction between the tip and sample. As described, the force curves are produced by measuring the vertical displacement and the tip deflection. The deflection measured by the photodiode is expressed in units of volts. Vertical tip deflection, $\delta$, can be related to the electrical signal, D, by:

$$
\delta^{\prime}=A\left(D-D_{0}\right)
$$

where $A$ is the cantilever sensitivity and $D_{0}$ is the initial deflection signal. Since the interaction is modeled as a spring, the deflection is directly related to the applied force, $\mathrm{P}$, by Hooke's Law. For the tip modeled as a spring:

$$
P=k \delta^{\prime}
$$

and for the sample:

$$
P=S\left(\delta-\delta^{\prime}\right)
$$

where $\mathrm{k}$ is the cantilever's spring constant, $\mathrm{S}$ is the contact stiffness, and $\delta$ is the vertical change in the piezo actuator. Combining these equations gives:

$$
\delta=A\left(D-D_{0}\right)(1+k / S)
$$

Relating this equation to the force curves shows that a plot of $\delta$ versus D has slope K:

$$
K=A(1+k / S)
$$

The contact stiffness is defined by Sneddon [17] as:

$$
S=2 E_{r} a
$$


where $\mathrm{E}_{\mathrm{r}}$ is the reduced modulus, and $a$ is the contact radius. By modeling the tip as a flat ended cylinder, a cantilever-tip property, $\alpha$, is defined:

$$
\alpha=k / 2 a
$$

substituting this into Equation 13 gives:

$$
K=A\left(1+\alpha / E_{r}\right) \quad E_{r}=\frac{\alpha}{K / A-1}
$$

This shows that if the cantilever sensitivity and cantilever-tip property can be found, the reduced modulus of the system can be found.

To find these two parameters, materials with known elastic moduli and Poisson's Ratios are used to calibrate the system. The reduced modulus can be found using Equation 17 for both calibration materials. With two solutions to Equation 17, the two unknowns $\alpha$ and A can be found. Then force curves can be collected on a sample with unknown properties, and the reduced modulus is found by using the slope. Equation 17 then relates the reduced modulus to the unknown sample:

$$
E_{r}=\frac{\left(1-v_{i}^{2}\right)}{E_{i}}+\frac{\left(1-v_{s}^{2}\right)}{E_{s}}
$$

where $E_{\mathrm{i}}$ and $E_{\mathrm{s}}$ are the indenter and sample moduli, respectively, and $v_{\mathrm{i}}$ and $v_{\mathrm{s}}$ are the Poisson Ratios. 


\section{Experimental Procedure}

\subsection{Samples}

To find the cantilever sensitivity and cantilever-tip property, two samples with known properties were used, a piece of a silicon wafer and polyethylene terephthalate (PET). In order to test the capability of the AFM as a nanoindenter, five unknown samples were tested in two separate experiments: nylon-12 and polystyrene (PS) were used for the first test; polymethyl methacrylate (PMMA), polycarbonate (PC), and low density polyethylene (LDPE) were used in the second test. The bulk polymer samples were mounted in epoxy and ground to expose a large surface to test. The samples were polished with silicon carbide paper down to 1200 grit. These were then polished with 6 $\mu \mathrm{m}$ diamond paste, $3 \mu \mathrm{m}$ alumina slurry, and finally a $.05 \mu \mathrm{m}$ alumina slurry. Each sample was ultrasonically cleaned between each stage of polishing.

\subsection{Nanoindentation}

In order to characterize the polymer samples, nanoindentations were performed using a MTS Nanoindenter XP. Nine indents were preformed on each of the polymer samples. The indentation depth was set to a maximum of $1000 \mathrm{~nm}$. The load method was identical for each sample except for the maximum load achieved. The indenter is held at a high load to determine the viscoelastic deformation, and then held at a low load to determine the thermal drift. The software can then correct for these parameters and calculate the hardness and elastic modulus of the samples. 


\subsection{AFM}

A Veeco Dimension 3000 AFM was used for the nanoindentation tests, using a Budget Sensors Tap300Al tip. This tip is made of silicon with an aluminum reflex coating, and has an estimated tip radius of $10 \mathrm{~nm}$. Two separate tests were run: the first tested nylon-12 and PS, the second tested PMMA, PC, and LDPE. Each test used a different probe, so the cantilever spring constant was found for each one. The spring constant for the first cantilever was found to be $29.83 \mathrm{~N} / \mathrm{m}$ and the second $31.30 \mathrm{~N} / \mathrm{m}$. The system was calibrated on the silicon sample to determine the deflection sensitivity for each test, which was kept constant for the indents on the polymers.

Each sample was scanned to determine the roughness of the surface and to avoid any contaminants that could affect the results. Once a suitable location was found, the probe was then pushed into the surface to carry out the indentation. To perform the indentation, the microscope was set into force calibration mode. In this mode, the tip is held stationary above the surface. The probe can be moved over the surface with $\mathrm{x}$ and $\mathrm{y}$ offsets allowing for the tests to be performed at specific locations. Fifteen indents were made at random locations on the surface using a scan rate of $2 \mathrm{~Hz}$. Each indent was separated by a space of $50 \mathrm{~nm}$ to avoid any changes in the local properties caused by an indentation affecting the results of the following indentation.

The vertical displacement and cantilever deflection are recorded throughout the indentation, producing a force curve for each indent made. After the indentations were completed the tip was withdrawn. This method does not create an indent that needs to be rescanned and measured. The force curves were then converted using SPIP software so that they could be analyzed. 


\section{$5 \quad$ Results and Discussion}

\subsection{Topography and Roughness}

Nanoindentation results can be affected by the condition of the sample surface. Peaks and valleys can influence how the tip contacts the surface, affecting the accuracy of the results. Prior to indentation, each sample was scanned with the AFM to obtain images of the topography and determine the roughness of the samples. Figure 7 shows representative images of each sample, along with cross section images in Figure 8. The five polymer samples show more prominent features than the silicon sample, most likely due to the polishing process. The parallel trenches seen in the PET sample are evidence of remaining polishing artifacts. The PS and PMMA samples also show some directionality (vertically in the images) which is also due to polishing. The nylon-12, PC, and PMMA have no apparent artifacts, but have much larger variations in topography. Since the silicon was fabricated and not polished like the polymer samples, it has the lowest roughness and has the most consistent topography of the samples. 


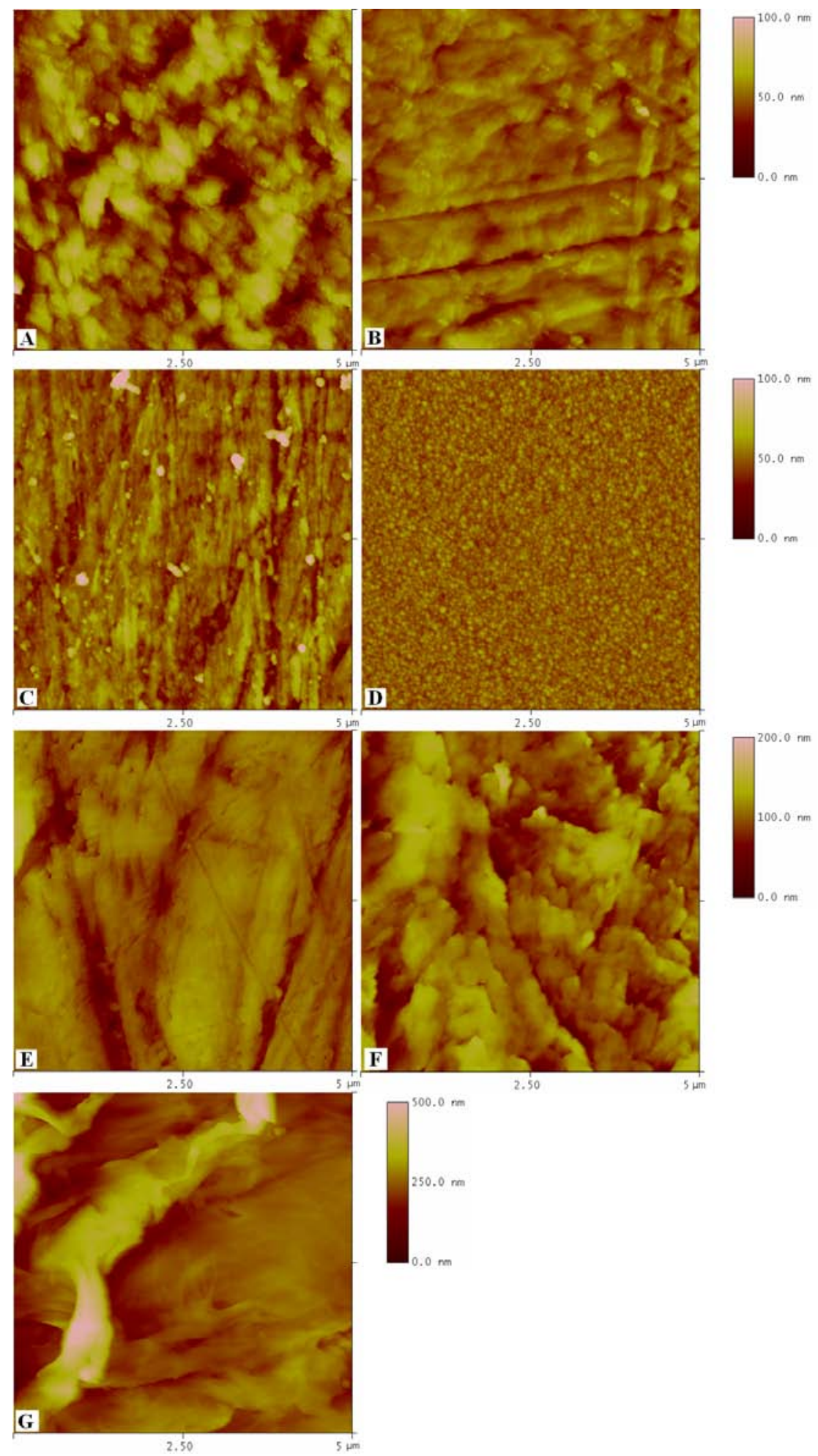

Figure 7: Topographic images of each sample taken prior to nanoindentation: A. Nylon-12; B. PET; C. PS; D. Silicon; E. PMMA; F. PC; G. LDPE. The features can affect the outcome of the test, so imaging the surface to find suitable test locations is important. 

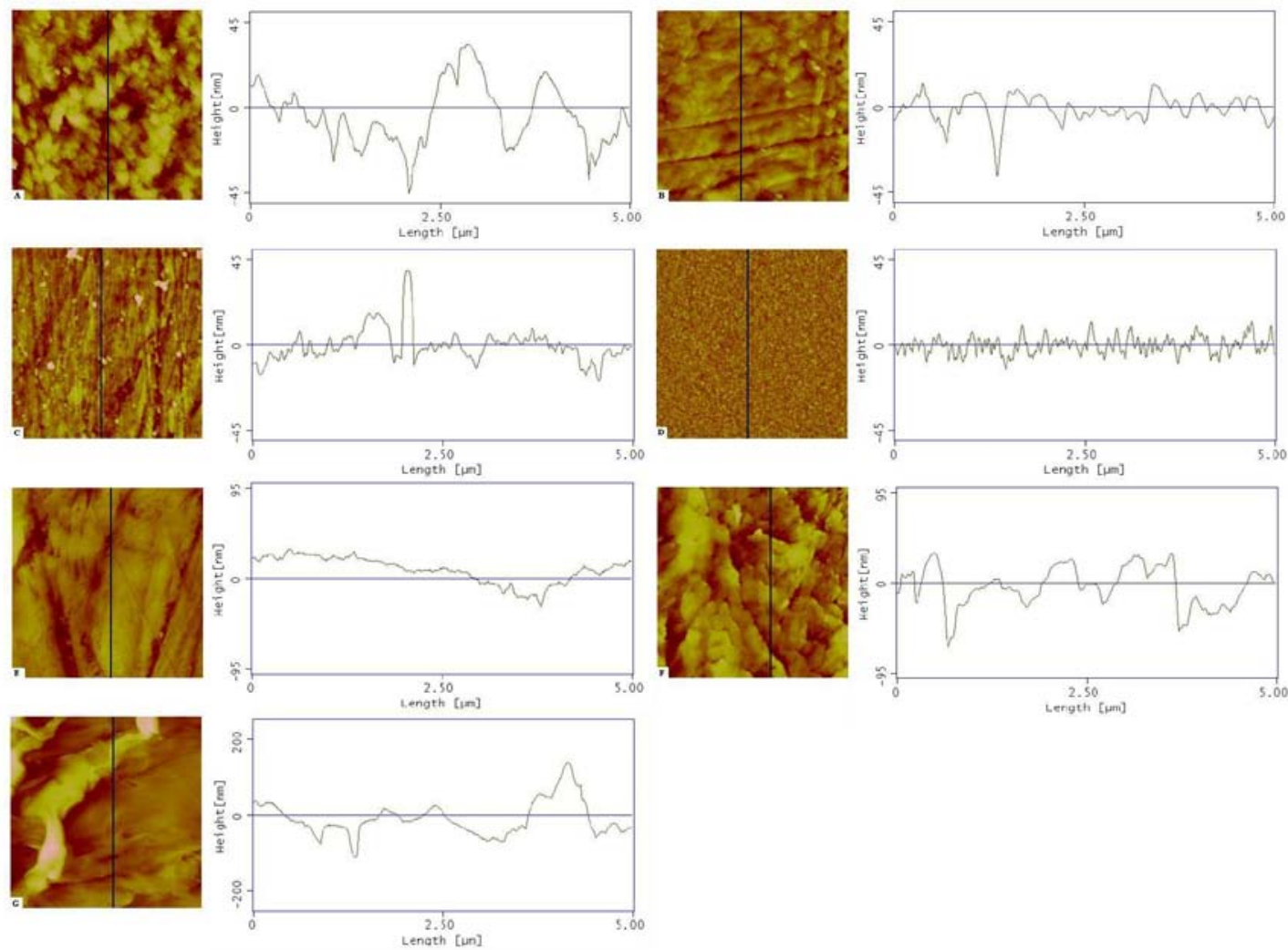

Figure 8: Cross sectional images of the sample surfaces. The dark line on the topographic images represents where the cross section was taken. Cross sections can help characterize surface features to determine how they may affect the nanoindentation.

Three different roughness parameters can be readily calculated with the AFM, the root mean squared ( $\left.\mathrm{R}_{\mathrm{RMS}}\right)$, arithmetic mean $\left(\mathrm{R}_{\mathrm{a}}\right)$, and the ratio of the image surface area to the projected surface area (r). The first two values can give information regarding the height of asperities while the third shows how much additional surface area there is compared to the two dimensional image. Table 1 shows the calculated roughness values for each sample. The cut and polished polymer samples are rougher than the silicon, since the silicon did not have to be polished. Most of the polymers have low roughness, but the soft LDPE sample did not polish as well and so is much rougher than the other 
samples. However since the large ridges and peaks were identified prior to testing they could be avoided, reducing the effect of the roughness on the elastic modulus calculations.

Table 1: Average roughness data taken from five images. Three roughness parameters are presented, root mean squared (RMS), arithmetic mean (Ra), and the ratio of the image surface area to the image projected surface area (r).

\begin{tabular}{|c|c|c|c|}
\hline & $\begin{array}{c}\text { RMS Roughness } \\
(\mathrm{nm})\end{array}$ & $\begin{array}{c}\text { Ra Roughness } \\
(\mathrm{nm})\end{array}$ & $\begin{array}{c}\text { r Roughness } \\
(\mu \mathrm{m} / \mu \mathrm{m})\end{array}$ \\
\hline Nylon-12 & $14.82 \pm 1.97$ & $11.58 \pm 1.57$ & $1.03 \pm 0.01$ \\
\hline PET & $10.33 \pm 3.73$ & $7.13 \pm 1.90$ & $1.02 \pm 0.01$ \\
\hline PS & $12.04 \pm 2.45$ & $8.22 \pm 1.54$ & $1.03 \pm 0.01$ \\
\hline PMMA & $13.43 \pm 2.42$ & $10.17 \pm 1.88$ & $1.02 \pm 0.01$ \\
\hline PC & $28.73 \pm 8.61$ & $21.10 \pm 4.79$ & $1.09 \pm 0.02$ \\
\hline LDPE & $118.07 \pm 62.69$ & $90.76 \pm 51.55$ & $1.26 \pm 0.13$ \\
\hline Silicon & $4.33 \pm 0.37$ & $3.40 \pm 0.23$ & $1.03 \pm 0.01$ \\
\hline
\end{tabular}

The presence of these peaks and valleys can affect the contact area between the tip and the sample during indentation, causing variations in the results. If the tip contacts on a peak, the indentation depth will be larger as compared to indenting a flat surface with the same load. The reverse is true if the tip contacts a valley, the indentation depth will be lower than on the flat surface [24]. Because of these effects, it is important to avoid these features when performing nanoindentation. The imaging capabilities of the AFM can be used to find suitable areas to test. In addition, the effects of peaks and valley can be reduced with the AFM due to the small tip size. The radius of the AFM tip is smaller than a typical nanoindenter tip. As a result, even if the AFM tip contacts on a protuberance, locally the surface may be flat and so the contact area may remain the same as the horizontal surface. 


\subsection{Mechanical Properties from Nanoindenter}

In order to determine the accuracy of the AFM results, each sample was first tested with traditional nanoindentation. Nine tests were performed on each polymer sample in order to reduce error due to roughness or creep effects. A sample loading curve can be seen in Figure 9. The nanoindenter includes holding segments in the loading and unloading phases to evaluate the viscoelastic creep and thermal drift. The software can then adjust the final results accordingly. Since silicon has well defined properties, it was not tested with nanoindentation. Values for its modulus and Poisson's ratio were instead found in a materials database.

Table 2 shows the elastic modulus results from the nanoindentation as well as the elastic moduli and Poisson's ratio's found in databases. The results for the polymer samples match well with the values found in the databases. Variations could be caused by the thermal and creep effects, or by the roughness of the samples as perviously discussed. Additionally, the mechanical properties of polymers can vary between samples due to their non-crystaline structure. 


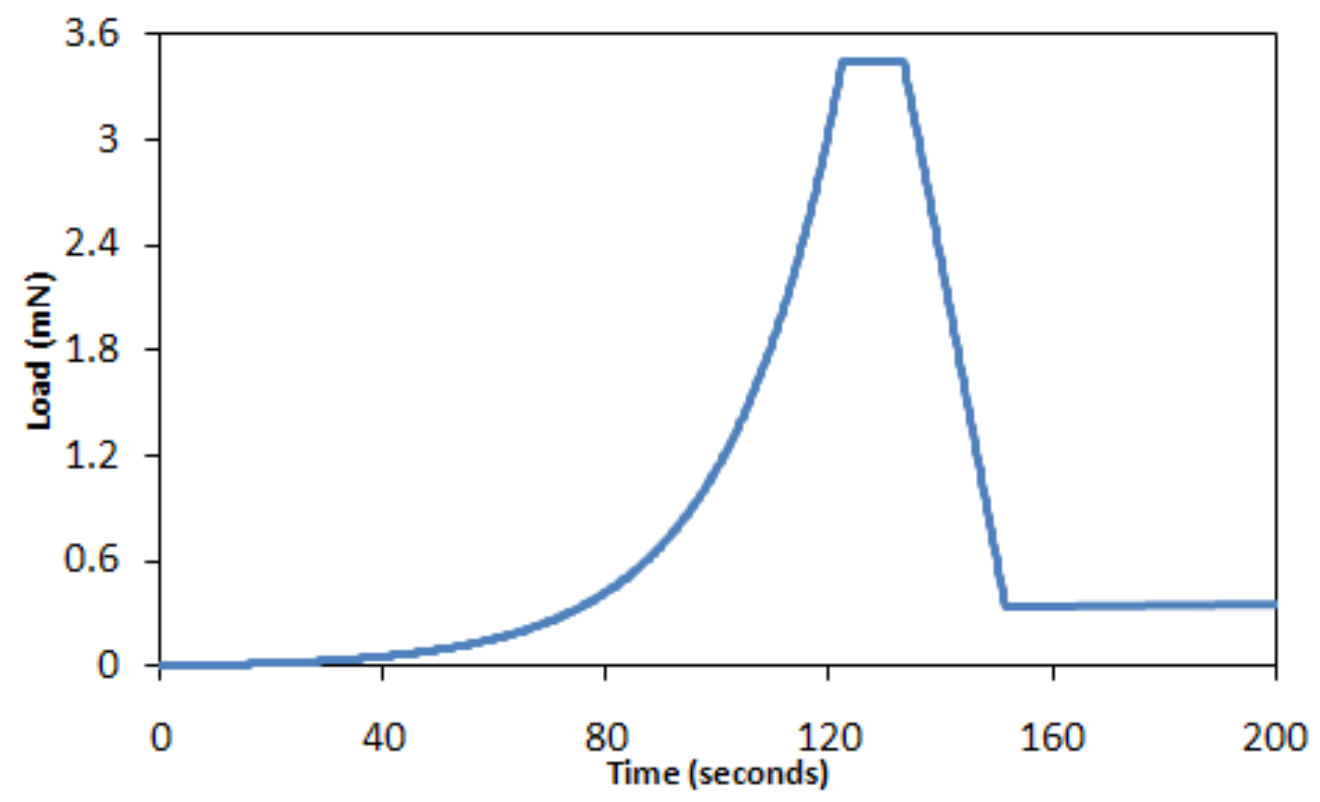

Figure 9: A sample loading curve showing the load schematic for the nanoindentation tests. This curve was taken from a PET test. The loading schemes for the other samples are similar except for the maximum load applied.

Table 2: Elastic modulus data from both the nanoindentation tests and from databases for comparison. The Poisson's ratio found for each sample is also given. These values were used in the final calculations.

\begin{tabular}{|c|c|c|c|}
\hline & $\begin{array}{c}\text { Elastic Modulus } \\
(\mathrm{GPa})\end{array}$ & $\begin{array}{c}\text { Elastic Modulus from } \\
\text { Database (GPa) }\end{array}$ & Poisson's Ratio \\
\hline Nylon-12 & $1.61 \pm 0.07$ & $1.40[25]$ & $0.40[25]$ \\
\hline PET & $3.25 \pm 0.14$ & $2-4[25]$ & $0.44[25]$ \\
\hline PS & $4.26 \pm 0.07$ & $3.43[25]$ & $0.34[25]$ \\
\hline PMMA & $5.54 \pm 0.05$ & $1.8-3.1[25]$ & $0.35-0.40[25]$ \\
\hline PC & $3.18 \pm 0.44$ & $2.36[25]$ & $.038-.042[27]$ \\
\hline LDPE & $0.24 \pm 0.11$ & $0.24[25]$ & $0.5[27]$ \\
\hline Silicon & -- & $112.40[26]$ & $0.28[28]$ \\
\hline
\end{tabular}




\subsection{Modulus from AFM}

To extract the modulus data from the force curves, the slope of the unloading curve is found. The linearity of the unloading curves suggests that the tip radius did not vary throughout the test (See Figure 10). This method assumes the tip is a flat punch cylinder in which the tip radius is constant, and this linearity of the unloading curves supports this assumption. The unloading curve for the LDPE does exhibit non-linearity. If the tip is penetrating deeper into the LDPE than the other samples it may be reaching a point on the tip that can no longer be modeled as cylinder. Images of one of the tips used can be seen in Figure 11.

Using the first 100 points of the unloading curve, linear regression was preformed to find the slope of each of the curves. The average of these values was then used in the final calculations. Representative curves can be seen in Figure 10. For the silicon sample, the loading and unloading curves are directly atop each other, and a sharp change in slope at the point of contact. This suggests that no deformation occurred during the test. Since the silicon was used as the sample with 'infinite hardness' to calibrate the AFM, this is an ideal result. The polymer samples show various degree of separation between the loading and unloading curves. This deviation can be caused by adhesion forces between the tip and the sample, which can be seen in the nylon and PMMA curves, or plastic deformation. The LDPE and PC show a significant difference between the approaching and retracting curves, with little to no adhesion force. This suggests that these samples experienced a considerable amount of plastic deformation during the test. Since this method assumes an elastic interaction, this may add a large error in the results. 

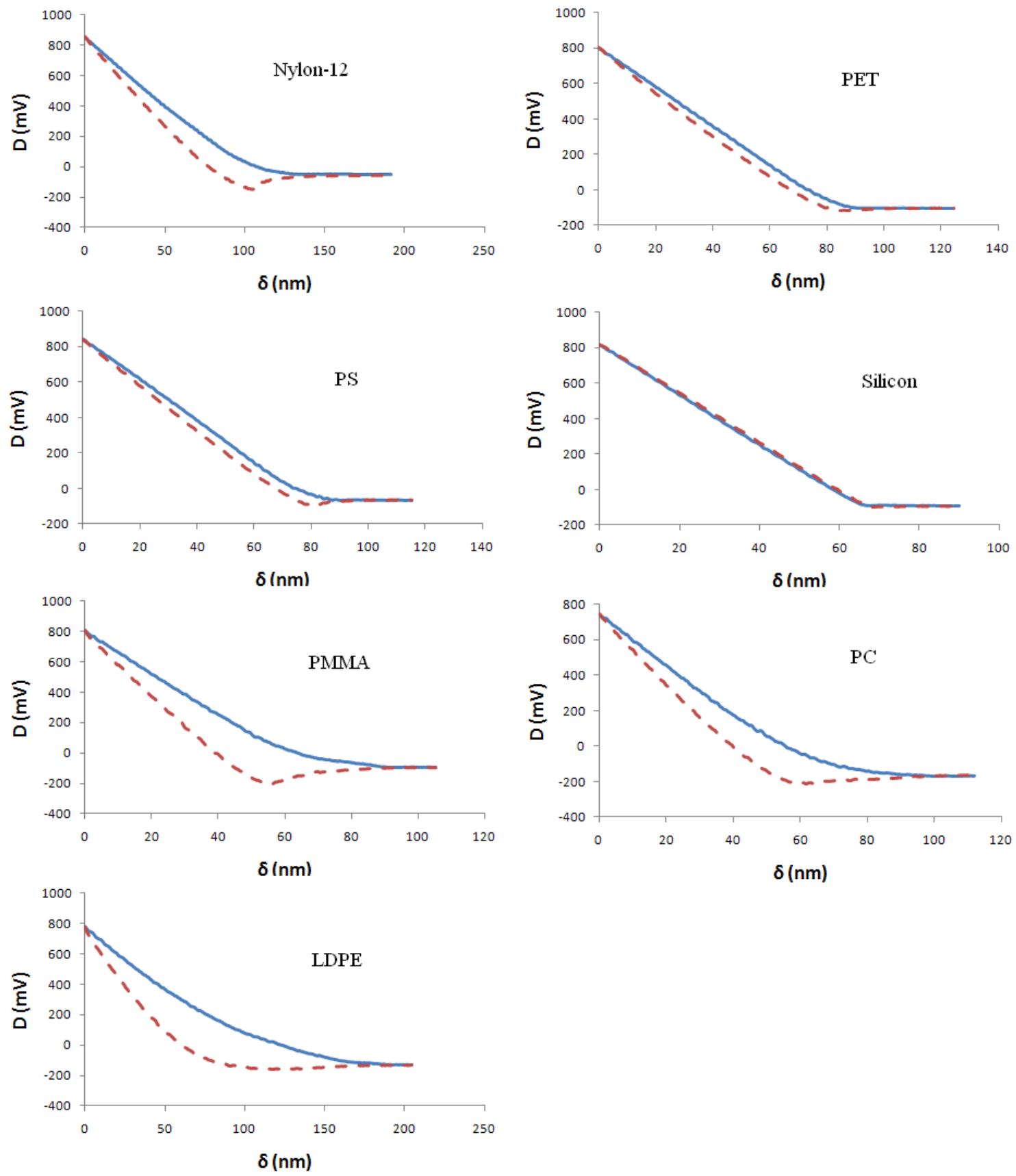

Figure 10: Representative distance versus deflection curves from the nanoindentation tests on the AFM. The solid lines represent the loading cycle while the dashed lines are the unloading cycle. 


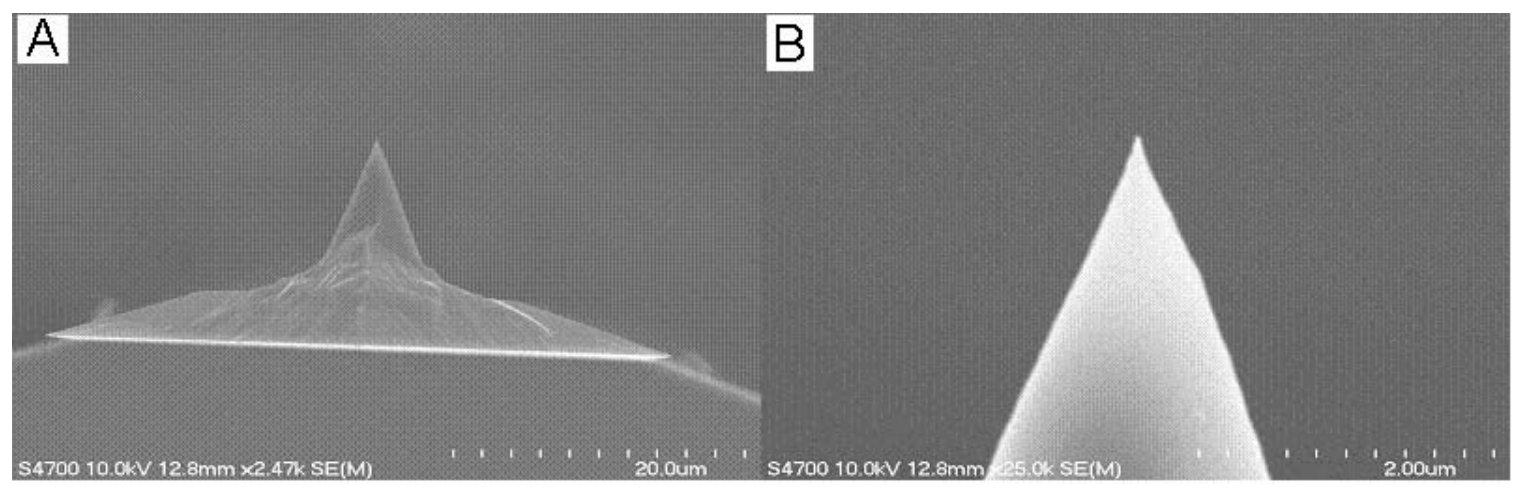

Figure 11: FESEM images of the tip used in the second set of tests. Image B shows a magnified picture of the tip. The tip radius from this image is about $50 \mathrm{~nm}$. The tip used in the first test was damaged and so the images were not included.

The K-values for the silicon and the PET are used to determine the values of $\alpha$ and $\mathrm{A}$ which were found as $0.31 \mathrm{GPa}$ and $72.5 \mathrm{~nm} \mathrm{nV}^{-1}$ respectively. Once these values were found, the K-values of the two unknowns were plugged into Equation 16 to find the reduced modulus. Then the elastic modulus of the unknowns could be found using Equation 17 and the Poisson's ratios in Table 2. The result of these calculations gives elastic moduli of $1.80 \mathrm{GPa}$ for the Nylon-12 and 5.05 GPa for the PS.

The same procedure was used for the other three polymers. Since a different tip was used, the K-values are not the same as the first test due to the different spring constant and tip radius. In this test the K-values for the silicon and PET were lower, due to the higher cantilever spring constant. The values for $\alpha$ and A were calculated as 0.26 GPa and $46.9 \mathrm{~nm} \mathrm{nV} \mathrm{n}^{-1}$ respectivly. Using these values, the elastic moduli for the remaining polymers were calculated and found to be: $6.35 \mathrm{GPa}$ for PMMA, 2.11 GPa for PC, and 0.36 GPa for LDPE. 
Table 3: Average K-values calculated from fifteen unloading curves for each sample. K-values are given in units of $\mathbf{n m ~} \mathbf{n V}^{-1}$.

\begin{tabular}{|c|c|c|}
\hline Sample & Test 1 & Test 2 \\
\hline Silicon & $72.9 \pm 1.20$ & $46.4 \pm 0.4$ \\
\hline Nylon-12 & $83.2 \pm 0.5$ & Not Tested \\
\hline PS & $76.6 \pm 5.0$ & Not Tested \\
\hline PET & $78.3 \pm 4.90$ & $50.2 \pm 2.6$ \\
\hline PMMA & Not Tested & $48.6 \pm 0.3$ \\
\hline PC & Not Tested & $51.9 \pm 0.7$ \\
\hline LDPE & Not Tested & $74.1 \pm 1.4$ \\
\hline
\end{tabular}

Table 4: Comparison between the elastic modulus results from the nanoindenter and the AFM.

\begin{tabular}{|c|c|c|c|}
\hline Sample & $\begin{array}{c}\text { Elastic Modulus } \\
\text { from Nanoindenter } \\
(\mathrm{GPa})\end{array}$ & $\begin{array}{c}\text { Elastic Modulus from } \\
\text { AFM (GPa) }\end{array}$ & Difference (\%) \\
\hline Nylon-12 & $1.61 \pm 0.07$ & $1.80 \pm 0.38$ & 11.80 \\
\hline PS & $4.26 \pm 0.07$ & $5.05 \pm 2.79$ & 18.54 \\
\hline PMMA & $5.54 \pm 0.05$ & $6.35 \pm 2.71$ & 14.62 \\
\hline PC & $3.18 \pm 0.44$ & $2.11 \pm 0.38$ & 33.65 \\
\hline LDPE & $0.24 \pm 0.11$ & $0.36 \pm 0.028$ & 50.0 \\
\hline
\end{tabular}

To determine if the nanoindenter results and the AFM results are statistically different, a t-test was performed for each sample. Using the average values and standard deviations in Table 4, t-statistics were calculated for each sample by:

$$
t=\frac{\mu_{1}-\mu_{2}}{\sqrt{\frac{\sigma_{1}^{2}}{N_{1}}+\frac{\sigma_{2}^{2}}{N_{2}}}}
$$


were $\mu_{\mathrm{i}}$ is the mean value of the elastic modulus, $\sigma_{\mathrm{i}}$ is the standard deviation, and $\mathrm{N}_{\mathrm{i}}$ is the number of tests. The values denoted with a 1 represent the nanoindenter, and 2 represents the AFM. To find the critical t- value the degrees of freedom were determined by:

$$
d f=\left(N_{1}-1\right)+\left(N_{2}-1\right)
$$

Nine tests were run with the nanoindenter and fifteen with the AFM, giving 22 degrees of freedom. The critical t-value for a two sided test at a 95\% confidence limit, found in a table of t-values, is 2.07. The t-tests determined that the nylon-12, PS, and PMMA were less than the critical t-value, thus the mean moduli are not statistically different. The PC and LDPE have larger differences between the nanoindenter and AFM values than the other samples, and the t-test determined that the means have a statistically significant variation. This is likely due to plastic deformation in these samples. This deformation could be corrected by using a cantilever with a lower spring constant on these softer samples.

Table 5: T-statistics for each sample compared with the critical t-value for a $\mathbf{9 5 \%}$ confidence limit.

\begin{tabular}{|c|c|}
\hline Sample & T-statistic \\
\hline $\begin{array}{c}\text { Critical t-value for 95\% } \\
\text { confidence limit }\end{array}$ & 2.07 \\
\hline Nylon-12 & -1.88 \\
\hline PS & -1.10 \\
\hline PMMA & -1.16 \\
\hline PC & 6.06 \\
\hline LDPE & -3.21 \\
\hline
\end{tabular}


In addition to plastic deformation, other issues may cause a difference between the two instruments. The MTS nanoindenter uses higher loads and deeper depths than the AFM. As a result, if there is any variation between the bulk modulus of the sample and the properties near the surface, it could appear as a deviation between the two tests. Additionally, the nanoindenter included hold periods to correct for viscoelastic deformation and thermal drift whereas the AFM tests did not. Since both of these have an effect in polymers, the AFM results may be affected more than the nanoindenter. Viscoelasic creep results in higher modulus values compared to bulk measurements, which may explain the higher value seen in the AFM results for the PS [14].

To check the accuracy of the tests, the cantilever-tip property can be used to estimate the tip radius. Using the spring constant, $\mathrm{k}$, from each test, and the calculated cantilever tip property, the respective tip radii can be calculated by $\alpha=k / 2 \mathrm{a}$. This results in a tip radius of $48.0 \mathrm{~nm}$ for the first probe and $59.2 \mathrm{~nm}$ for the second. The manufacturer give an estimated tip radius of $10 \mathrm{~nm}$, however, at this scale such an estimate is not always reliable. It is also possible that the tip was deformed or contaminated during the test, resulting in a larger radius. The calculated cantilever sensitivity A can also be checked. This value is defined as the slope of a $\delta$ versus D curve on a material of 'unlimited hardness.' In this test the silicon was used as this sample, therefore the calculated A value and the K-value from the silicon should be the same. From Table 3 the K-values from the silicon tests were $72.9 \mathrm{~nm} \mathrm{nV}^{-1}$, and $46.4 \mathrm{~nm}$ $\mathrm{nV}^{-1}$ compared to the calculated A values of $72.5 \mathrm{~nm} \mathrm{nV}^{-1}$ and $46.9 \mathrm{~nm} \mathrm{nV}$. The experimental and calculated values match well for both tests, indicating that the calculated values are accurate. 


\section{Conclusion}

A new method for determining the mechanical properties of a material by nanoindentation with an AFM was tested. This method uses two reference samples with known properties to calculate the cantilever sensitivity and a new value, the cantilever-tip property. This method reduces the need to attempt to measure these values directly, and thus is faster and easier than previous methods.

In this paper, five polymer samples were tested in two separate experiments to determine the accuracy of this method. Traditional depth-sensing nanoindentation was preformed to obtain values for comparison. Before AFM nanoindentation, the samples were scanned to determine the surface characteristics. The effects of surface feature on the indentation results was explored, and it was seen that the AFM has an advantage to traditional methods since it can be used to find suitable areas to test, and the smaller tip radius can lessen the effects of surface features.

Each sample was then indented with the AFM, and using this new method the elastic modulus was calculated. The results from the AFM tests for three of the five samples were shown to be statistically similar to the nanoindenter results, while the PC and LDPE were statistically different. This is likely due to plastic deformation in these samples. To further check the calculations, the cantilever-tip property was used to estimate the tip radius. The results of $48.0 \mathrm{~nm}$ and $59.2 \mathrm{~nm}$ were higher than the manufacturer's value of $10 \mathrm{~nm}$, but this is most likely due to wearing and blunting of the tip during nanoindentation and imaging. The calculated cantilever sensitivities were compared to the K-values from the nanoindentation of the silicon. These values were seen to be nearly identical, confirming that this calculation was accurate. With these two 
checks, and the comparison with the nanoindenter results, it can be said that the new method for finding the elastic modulus is reliable and accurate.

The nano-scale imaging capabilities, combined with the ability to perform nanoindentation tests, make the AFM a useful tool of characterizing materials on the nanometer scale. Sample surfaces can be imaged before testing to find suitable test sites, or to identify nano-structures for testing. The AFM can also be used to find different phases in a sample, allowing for accurate testing of a multiphase material. Additionally, the low load and shallow indentation depth of AFM nanoindentation make it suitable for thinner samples, such as coatings and thin films.

Since this method is proven reliable on bulk materials, future work can test it on more specific sample types. This technique could be used to better understand the nanoscale properties of materials, benefiting fields such as biology, chemistry, and materials science. Future testing could include experiments involving multiphase systems, thin films, or nanostructures. This method could also potentially be expanded beyond polymer systems. Tests on stiffer materials such as metals could be performed if a stiffer cantilever is used. Expanding upon these techniques could allow for the nanoscale properties of many different material types to be accurately studied. 


\section{$7 \quad$ References}

1. M. R. VanLandingham, S. H. McKnight, G. R. Palmese, J. R. Elings, X. Huang, T. A. Bogetti, R. F. Gillespie. "Nanoscale Indentation of Polymer Systems Using the Atomic Force Microscope.” The Journal of Adhesion. 1997; 64(1-4): 31-59.

2. D. Passeri, A. Bettucci, A. Biagioni, M. Rossi, A. Alippi, E. Tamburri, M. Lucci, I. Davoli, and S. Berezina. "Indentation Modulus and Hardness of Viscoelastic Thin Films by Atomic Force Microscopy: A Case Study.” Ultramicroscopy. 2009; 109: 1417-1427.

3. G. L. Hornyak, H. F. Tibbals, J. Dutta, J. J. Moore. Introduction to Nanoscience and Nanotechnology. (Taylor and Francis Group, 2009).

4. M. F. Doerner, and W. D. Nix. “A Method for Interpreting the Data from DepthSensing Indentation Instruments.” Journal of Materials Research. 1986; 1(4): 601-609.

5. W. C. Oliver, G. M. Pharr. “An Improved Technique for Determining Hardness and Elastic Modulus Using Load and Displacement Sensing Indentation Experiments.” Journal of Materials. 1992; 7(6): 1564-1583.

6. X. Chen, and J. J. Vlassak. "Numerical Study on the Measurment of Thin Film Mechanical Properties by Means of Nanoindentation.” Journal of Materials Research. 2001; 16(10): 2974-2982.

7. B. Bhushan, and V. N. Konikar. "Nanoindentation Hardness Measurements Using Atomic Force Microscopy.” Applied Physics Letters. 1994; 64(13): 1653-1655.

8. D. Tranchida, S. Piccarolo, and M. Soliman. "Nanoscale Mechanical Characterization of Polymers by AFM Nanoindentations: Critical Approach to the Elastic Characterization.” Macromolecules. 2006; 39: 4547-4556.

9. R. Bassani, R. Solaro, M. Alderighi, C. Di Cesare, and M. Allegrini. "Nanoindentation With AFM.” International Conference on Tribology. 2006

10. B. Tang, A. H. W. Ngan, and J. B. Pethica. “A Method to Quantitatively Measure the Elastic Modulus of Materials in Nanometer Scale Using Atomic Force Microscopy.” Nanotechnology. 2008; 495713 (7pp).

11. D. L. Hiemstra, and N. R. Sottos. "Thermally Induced Interfacial Microcracking in Polymer Matrix Composites.” Journal of Composite Materials. 1993; 27(10): 1030-1051. 
12. M. Roy, J. K. Nelson, R. K. MacCrone, L. S. Schadler, C. W. Reed, R. Keefe, and W. Zenger. "Polymer Nanocomposite Dielectrics - The Role of the Interface." IEEE Transactions on Dielectrics and Electrical Insulation. 2005; 12(4): 629-643.

13. J. V. Ernst. "Nanotechnology Education: Contemporary Content and Approaches." The Journal of Technology Studies. 2009; 35(1): 3-8.

14. Mark R. VanLandingham, John S. Villarrubia, William F. Guthrie, and Greg F. Meyers. "Nanoindentation of Polymers: An Overview." Macromolecular Symposia. 2001; pg 15-43.

15. Anthony C. Fisher-Cripps. Introduction to Contact Mechanics, $2^{\text {nd }}$ Edition. Springer Science+Business Media LLC. 2007; pg 189-199.

16. J.L. Meneve, J.F. Smith, N.M. Jennett, and S.R.J. Saunders. "Surface Mechanical Property Testing by Depth Sensing Indentation.” Applied Surface Science. 1996; 100/101: 64-68.

17. I. N. Sneddon. "The Relation Between Load and Penetration in the Axisymmetric Boussinesq Problem for a Punch of Arbitrary Profile.” International Journal of Engineering Science. 1965; 3(1): 47-57.

18. A. Bolshakov, and G. M. Pharr. "Influences of Pileup on the Measurement of Mechanical Properties by Load and Depth Sensing Indentation Techniques." Journal of Materials Research. 1998; 3(4): 1049-1058.

19. K. W. McElhaney, J. J. Vlassak, and W. D. Nix. "Determination of Indenter Tip Geometry and Indentation Contact Area for Depth Sensing Indentation Experiments.” Journal of Materials Research. 1998; 13(5): 1300-1306.

20. G. Binning, H. Rohrer, Ch. Gerber, and E. Weibel. "Surface Studies by Scanning Tunneling Microscopy.” Physical Review Letters. 1982; 49(1): 57-60.

21. Digital Instruments. Scanning Probe Microscopy Training Notebook, Version 3.0. Digital Instruments Veeco Metrology Group. 2000.

22. J. S. Villarrubia. "Algorithms for Scanned Probe Microscope Image Simulation, Surface Restoration, and Tip Estimation.” Journal of Research of the National Institute of Standards and Technology. 1997; 102(4): 425-454.

23. M. R. VanLandingham, T. F. Juliano, M. J. Hagon. "Measuring Tip Shape for Instrumented Indentation Using Atomic Force Microscopy.” Measurement Science and Technology. 2005; 16: 2173-2185. 
24. K.-D. Bouzakis, N. Michailidis, S. Hadjiyannis, G. Skordaris, and G. Erkens. "The Effect of Specimen Roughness and Indenter Tip Geometry on the Determination Accuracy of Thin Hard Coatings Stress-Strain Laws by Nanoindentation ." Materials Characterization.2002; 49(2): 149-156.

25. B. Ellis, and R. Smith (Eds), Polymers: A Property Database $2^{\text {nd }}$ Edition. CRC Press, Boca Raton, FL (2008).

26. Metals Handbook, Vol.2 - Properties and Selection: Nonferrous Alloys and SpecialPurpose Materials, $10^{\text {th }}$ Edition. American Society for Metals (1990)

27. G. Erhard, Designing with Plastics, Verlag, Munich, Germany (2006).

28. William D. Callister, Fundamentals of Materials Science and Engineering, $2^{\text {nd }}$ Edition. John Wiley and Sons Inc. Hoboken, NJ (2005). 